\title{
IMPACTO DE LA ALTURA EN EL EMBARAZO Y EN EL PRODUCTO DE LA GESTACIÓN
}

\author{
Gustavo F. Gonzales $1,2, a$
}

\begin{abstract}
RESUMEN
Esta revisión describe los procesos asociados con mantener un embarazo en mujeres nacidas al nivel del mar que cursan su embarazo en la altura; en aquellas que viajan intermitentemente, o las que nacen y residen en la altura. La literatura examinada demuestra que la frecuencia de malformaciones congénitas es elevada en la altura; que las tasas de mortalidad fetal tardía, nacidos pequeños para su edad gestacional, y preeclampsia se encuentran incrementadas en la altura, asociadas a valores altos de hemoglobina materna (>14,5 g/dL). En conclusión, el embarazo en una mujer expuesta de forma aguda, intermitente o permanente a las grandes alturas, genera mayores riesgos en comparación con un embarazo desarrollado a nivel del mar.
\end{abstract}

Palabras clave: Altitud; Embarazo; Muerte fetal; Edad gestacional; Preeclampsia; Niño (fuente: DeCS BIREME).

\section{IMPACT OF HIGH ALTITUDE ON PREGNANCY AND NEWBORN PARAMETERS}

\begin{abstract}
This review describes adverse outcomes in pregnancy after brief, intermittent, or permanent residence at high altitudes. Review of literature shows that congenital malformations rates are higher at high altitudes. Additionally, rates of stillbirths, small size for gestational age, and preeclampsia are increased in populations living at high altitudes and are associated with high maternal hemoglobin levels (>14.5 g/dl). In conclusion, a pregnant woman exposed briefly, intermittently, or permanently to high altitudes results in increased risk of adverse outcomes when compared to pregnancies observed at sea level.
\end{abstract}

Key words: Altitude; Pregnancy; Fetal death; Gestational age; Pre-eclampsia; Child (source: MeSH NLM).

\section{INTRODUCCION}

Se considera que una persona se ha adaptado a la altura cuando mantiene su capacidad de reproducción; cuando el recién nacido y la madre no afectan su salud por el embarazo; y las poblaciones en general, son capaces de practicar actividad física sin que se afecte su salud. La falta de adaptación a la altura se conoce como mal de montaña crónico.

Hace 40000 años, la mayor parte del mundo ya estaba poblado por la especie humana a excepción de Australia y América. Existe consenso en afirmar que los americanos descienden de los asiáticos quienes arribaron al final del pleistoceno (hace aproximadamente 30000 a 40000 años) a través del estrecho de Bering ${ }^{(1)}$.

Frente a los tibetanos, que han residido por más de 25000 años en los Himalayas, los pobladores de las zonas rocosas del Colorado, en Estados Unidos, con más de 300 años; y la etnia china han en los Himalayas desde hace 60 años, la antigüedad del poblador andino peruano (12 000 años) se catalogaría como intermedia, en razón de las evidencias arqueológicas ${ }^{(2)}$.

Con la llegada española en el siglo XVI, en el Perú ocurre un importante mestizaje cuyo efecto sobre la adaptación a la altura no se conoce aún con precisión. Existen evidencias que las poblaciones con menos mestizaje hispano como las que existen en los Andes sur tienen mayor tiempo de residencia generacional que las poblaciones en los Andes centrales ${ }^{(3)}$. De igual forma, se ha documentado que en una misma localidad hay pobladores con diferentes periodos de residencia generacional. Así, en Cerro de Pasco a 4340 m de altitud, las mujeres gestantes que tienen más de tres periodos generacionales en la altura presentan mejor saturación arterial de oxígeno e hijos con mayor peso al nacer ${ }^{(4)}$.

Las mujeres en las alturas de Perú se caracterizan por conservar su capacidad reproductiva ${ }^{(5)}$; sin embargo,

\footnotetext{
Unidad de Reproducción Humana, Instituto de Investigaciones de la Altura, Universidad Peruana Cayetano Heredia. Lima, Perú.

2 Departamento de Ciencias Biológicas y Fisiológicas, Facultad de Ciencias y Filosofía, Universidad Peruana Cayetano Heredia. Lima, Perú.

a Médico doctor en Ciencias
}

Recibido: 29-11-11 Aprobado: 18-04-12 
existen varios parámetros reproductivos que difieren con las mujeres residentes a nivel del mar: la menarquia es más tardía ${ }^{(6)}$ y la menopausia es más temprana ${ }^{(7)}$. En mujeres en edad reproductiva, los niveles plasmáticos de estradiol, progesterona ${ }^{(8)}$ y prolactina ${ }^{(9)}$ son menores en la altura que a nivel del mar, mientras que sucede lo contrario al controlar los niveles séricos de la hormona folículo estimulante durante la perimenopausia ${ }^{(10)}$. La presente revisión intenta mostrar el impacto que tiene la exposición a la altura en el curso de un embarazo.

\section{LA HISTORIA}

El efecto de la altura sobre la gestación se conoce desde hace mucho y fueron referidos por los cronistas españoles en la conquista al Perú. Durante la colonia, el padre Antonio de la Calancha (11) en su "Crónica moralizadora de la Orden de San Agustín" remarcaba que en la ciudad de Potosí (4067 m de altitud) aunque el nativo de altura se reproducía normalmente, los españoles afincados no pudieron tener descendencia sino hasta 53 años después de su llegada, atribuyendo el primer nacimiento de un criollo ocurrido en 1598, a un milagro de San Nicolás de Tolentino.

Martínez Vela lo refirió de la siguiente manera: Don Francisco Flores y doña Leonor de Guzmán tuvieron seis hijos, más ninguno le vivió. Un día el Padre Prete les dijo que se encomendaran a San Nicolás de Tolentino, siguió el consejo y el día de la Navidad parió un niño muy hermoso. Fue el primero que se logró (sobrevivió) que los que en Potosí nacieron ${ }^{(12)}$.

La menor supervivencia de los hijos de españoles en zonas altoandinas podría estar asociada con una falta de adaptación, lo cual no ocurría con los nativos ${ }^{(2)}$, y así se aprecia hasta la actualidad ${ }^{(13)}$. Los nativos de altura, luego de una estancia mayor a 10000 años, pueden reproducirse, y sus hijos sobrevivir de manera adecuada en las alturas; en tanto, en aquellos que se exponen temporalmente, la mortalidad infantil es muy alta ${ }^{(2)}$

El padre Bernabé Cobo, jesuita español (1653) en su libro "Historia del nuevo mundo" detalló cómo los hijos de los nativos sobrevivían en la altura a diferencia de los hijos de los españoles. Él señala que este es un fenómeno asociado al tipo de herencia de cada individuo; afirma que: A más cuarterones de sangre hispana del recién nacido su chance de morir es mayor, en tanto que a más cuarterones de sangre nativa, la posibilidad de sobrevivir aumentaba. Los cuarterones se refieren al origen de los cuatro abuelos que tiene cada persona, y que puede fluctuar desde cuatro abuelos españoles hasta cuatro abuelos nativos ${ }^{(14)}$.

\section{LA EVIDENCIA CIENTIIFICA}

Se estima que más de 140 millones de personas habitan permanentemente en alturas mayores a $2500 \mathrm{~m}$ de altitud ${ }^{(15)}$, nivel que corresponde a una presión arterial de oxígeno $\left(\mathrm{PaO}_{2}\right)$ de 60 a $70 \mathrm{mmHg}$, punto en el cual la saturación arterial de oxígeno empieza a disminuir exponencialmente según la caída de la $\mathrm{PaO}_{2}{ }^{(16)}$. La altura se convierte, por ello, en un inmenso laboratorio natural donde se puede estudiar el impacto de la hipoxia. Los estudios de investigación han demostrado que el recién nacido en la altura es de menor peso y que la magnitud de la reducción es inversamente proporcional con el número de generaciones de ancestros con residencia en la altura ${ }^{(3,17)}$.

En los últimos años se ha clarificado que este menor peso al nacer se debe a una restricción en el crecimiento intrauterino $^{(16,18,19,20)}$. Este menor crecimiento intrauterino, evidente a partir de las 20 semanas de gestación, es por menor flujo arterial útero-placentario (21) que, a su vez, se asocia con un incremento en el hematocrito/ hemoglobina, que conduce a un estado de mayor viscosidad sanguínea ${ }^{(22)}$. También se ha planteado una menor disponibilidad de glucosa como causa de esta restricción ${ }^{(23)}$. Esto se puede asociar con la baja tasa de diabetes gestacional en la altura ${ }^{(24)}$.

El feto a nivel del mar experimenta un grado de hipoxemia que es similar al observado en adultos que habitan entre 4000 y 5000 m de altitud, con una presión parcial de la vena umbilical de oxígeno de $50 \mathrm{mmHg}{ }^{(25)}$. Aun así, hay evidencia indirecta que el feto en la altura es más hipóxico que a nivel del mar, ello se basa en el hallazgo en una población boliviana a $3600 \mathrm{~m}$ de altitud, donde el hematocrito/hemoglobina fetal es mayor que en Santa Cruz (400 m de altitud) ${ }^{(26)}$.

Es evidente que el grado de hipoxia fetal va a depender de múltiples causas. En La Paz, las gestantes con ascendencia andina tienen mayor flujo de la arteria uterina, marcador indirecto del flujo útero-placentario, que las de ascendencia europea (21). Asimismo, ellas presentan menor hematocrito/hemoglobina, y mayor peso del recién nacido que las descendientes de europeas, a pesar de estar viviendo ambos grupos bajo el mismo nivel de altitud (3600 m de altitud) ${ }^{(16)}$.

En ese sentido, el aumento del hematocrito/hemoglobina materna es un factor importante que indirectamente favorecelageneración de hipoxia en el feto. Recientemente se ha demostrado que la restricción en el crecimiento intrauterino es una situación asociada con el incremento en los niveles de hemoglobina, tanto a nivel del mar como en la altura moderada ${ }^{(18)}$, o en la gran altitud ${ }^{(20)}$. 
De acuerdo con diversos estudios en distintas poblaciones de altura, la reducción en el crecimiento intrauterino es un efecto per se de la altura y no asociado con el nivel socioeconómico ${ }^{(3,27)}$. Esto no descarta que el nivel socioeconómico contribuya, de manera adicional, en modificar la tasa de restricción en el crecimiento intrauterino.

\section{HIPOXIA Y DESARROLLO PLACENTARIO}

Las primeras semanas de la gestación se producen en un medio hipóxico donde las glándulas histiotróficas dan nutrición al feto hasta las diez semanas ${ }^{(28)}$ proveyendo nutrientes en condiciones de baja concentración de oxígeno ${ }^{(29)}$. Aunque parece ser necesario un medio hipóxico para evitar el daño oxidativo producido por el oxígeno ${ }^{(28)}$, evidencias recientes refieren que la hipoxemia en la altura afecta la embriogénesis y, por ende, la organogénesis ${ }^{(19)}$.

Según estudios de ultrasonografía doppler, entre la semana diez y doce empieza el flujo sanguíneo al espacio intervelloso ${ }^{(30)}$. Cuando se ha completado la organogénesis, hay una transición a la nutrición hemotrófica al inicio del segundo trimestre, cuando la circulación placentaria se ha establecido totalmente ${ }^{(31)}$. En este momento la tensión de oxígeno de la placenta se incrementa en tres veces ${ }^{(32)}$.

El estrés oxidativo en esta etapa, contribuye a la preeclampsia y a los abortos ${ }^{(33)}$. De igual modo, la hipoxia de la altura afecta esta etapa del desarrollo fetal donde ocurre la organogénesis. Por ello, la tasa de anomalías congénitas en las poblaciones peruanas por encima de $3500 \mathrm{~m}$ de altitud son cuatro veces más altas que a nivel del mar (2) (Tabla 1). Esto mismo se observa en otras poblaciones de altura ${ }^{(34-36)}$.

\section{HIPOXIA Y RESTRICCIÓN DEL CRECIMIENTO FETAL}

En la semana 23, tras el mayor crecimiento de la placenta, se inicia el mayor desarrollo del feto (19). Estudios con ultrasonido doppler a 3600 m de altitud en Bolivia, encontraron diferencias en el flujo de la arteria

Tabla 1. Malformaciones congénitas a nivel del mar y en la altura del Perú ${ }^{(2)}$.

\begin{tabular}{lccc}
\hline Lugar & $\begin{array}{c}\text { Número de } \\
\text { recién nacidos }\end{array}$ & $\begin{array}{c}\text { Malformaciones } \\
\text { congénitas }\end{array}$ & $\begin{array}{c}\text { Frecuencia } \\
(\%)\end{array}$ \\
\hline A nivel del mar & 14029 & 137 & 0,98 \\
\hline Altura $>3500 \mathrm{~m}$ & 10443 & 408 & 3,91 \\
\hline
\end{tabular}

uterina desde las 20 semanas de gestación ${ }^{(21)}$. Al aplicar la misma metodología se ha podido determinar que la diferencia en el tamaño fetal, entre Cerro de Pasco (4320 m de altitud) y Lima (150 m de altitud), se observa a partir de las 25-29 semanas de gestación ${ }^{(37)}$.

Evaluando los partos, en tres ciudades de altura como Huancayo (3280 m de altitud), Cusco (3400 m de altitud) y Juliaca (3800 $\mathrm{m}$ de altitud), el peso del recién nacido se hace menor con respecto a Lima (150 m de altitud) a partir de las 34 semanas de gestación ${ }^{(38)}$. Estos datos indican que la restricción del crecimiento se hace evidente desde el segundo trimestre y continúa en el tercer trimestre del embarazo.

Recientemente, se ha demostrado que un incremento en los niveles de hemoglobina por encima de $13,5 \mathrm{~g} / \mathrm{dL}$ y más aun sobre $14,5 \mathrm{~g} / \mathrm{dL}$ (eritrocitosis) reduce el crecimiento fetal tanto a nivel del mar como en moderada o gran altitud ${ }^{(18,20)}$.

\section{MORTALIDAD FETAL TARDÍA Y MORTALIDAD NEONATAL}

La mortalidad fetal tardía es la muerte después de 28 semanas o más ${ }^{\left({ }^{39)}\right.}$. Según la literatura científica, el riesgo es 4,82 veces mayor en la altura que a nivel del mar ${ }^{(31)}$.

En la Tabla 2 se observan datos obtenidos por Passano en Puno a $3800 \mathrm{~m}$ de altitud ${ }^{(13)}$. Se calculó la tasa de mortalidad fetal tardía (por mil nacidos vivos) y la tasa de mortalidad neonatal (antes de los 28 días de edad). La población fue clasificada como migrantes de altitudes menores a $3000 \mathrm{~m}$ de altitud (nacionales o extranjeras), nativos de Puno de zonas urbanas, nativos rurales aymaras y nativos rurales quechuas.

Tabla 2. Tasa de mortalidad fetal tardía y mortalidad neonatal en Puno (3800 m de altitud) en 7408 partos de acuerdo con el lugar de origen de la madre ${ }^{(13)}$.

\begin{tabular}{lcc}
\hline Origen & $\begin{array}{c}\text { Tasa de } \\
\text { mortalidad fetal } \\
\text { tardía x 1000 NV }\end{array}$ & $\begin{array}{c}\text { Tasa de } \\
\text { mortalidad } \\
\text { neonatal x 1000 NV }\end{array}$ \\
\hline $\begin{array}{l}\text { Migrantes de alturas } \\
\text { menores a 3000 m } \\
\text { de altitud }\end{array}$ & 51,52 & 39,39 \\
$\begin{array}{l}\text { Nativa de zonas } \\
\text { urbanas de Puno }\end{array}$ & 32,21 & 13,70 \\
$\begin{array}{l}\text { Nativa de zonas } \\
\text { rurales aymaras }\end{array}$ & 21,69 & 16,93 \\
$\begin{array}{l}\text { Nativa de zonas } \\
\text { rurales quechuas }\end{array}$ & 28,63 & 15,85 \\
\hline \begin{tabular}{l} 
Total \\
\hline
\end{tabular} & 29,29 & 16,33 \\
\hline
\end{tabular}

NV: nacidos vivos. 
Las tasas más altas de mortalidad fetal tardía y mortalidad neonatal ocurrieron en mujeres migrantes de zonas de baja altitud y que tuvieron su embarazo en Puno (3800 m de altitud). Estos datos sugieren, conforme refieren las crónicas de la conquista, que la migración de mujeres de una zona de menor a mayor altitud, puede llevar a un resultado fatal (2).

La mortalidad fetal tardía está fuertemente asociada con hipoxia y restricción del crecimiento intrauterino (40,41), dos factores que cohabitan en las madres expuestas temporalmente o tienen pocas generaciones residentes en la altura.

La mortalidad perinatal (hasta los siete días de nacido) y neonatal (primeros 28 días de nacido) es elevada en la altura. Este efecto es conocido desde hace muchos años y sus tasas elevadas se mantienen hasta la actualidad (Tabla 2) ${ }^{(2)}$. Siendo evidente que las poblaciones rurales son de menor nivel socioeconómico que las de zonas urbanas, el estudio de Passano demuestra que la tasa de mortalidad fetal tardía es mayor en nativos de zonas urbanas de Puno que en aymaras de zonas rurales ${ }^{(13)}$.

Cuando se estudia la mortalidad infantil (muertes hasta el año de vida) en poblaciones con necesidades básicas insatisfechas, se observa que la tasa de mortalidad Infantil es mayor en medio rural que en urbano, incrementándose a medida que aumenta la altitud de residencia ${ }^{(3)}$.

\section{MALFORMACIONES CONGÉNITAS}

Según una investigación realizada en 41 ciudades de Sudamérica, se encontró mayor número de malformaciones congénitas en tres ciudades por encima de los $2000 \mathrm{~m}$ de altitud. Las más frecuentes fueron: labio leporino; microtia (oreja pequeña); apéndice preauricular; anomalía de los arcos branquiales; síndrome de banda de constricción congénita, y atresia anal ${ }^{(42)}$. Cabe destacar que estas anomalías se desarrollan en el primer trimestre de gestación ${ }^{(43,44)}$. La vida en las grandes alturas está asociada con deformidad amniótica, adhesión y mutilación (síndrome ADAM) que puede deberse al defecto de las células germinales, disrupción vascular y alteraciones en los morfógenos durante la gastrulación temprana, conduciendo a anomalías congénitas ${ }^{(36)}$.

Durante los años ochenta se reportaron varios casos de craniosinostosis en las comunidades residentes en las alturas del estado de Colorado (EEUU). Esta es una alteración donde se produce el cierre prematuro de una o más de las suturas que separan los huesos del cráneo de un bebé. El riesgo de esta malformación fue 1,4 veces más en alturas mayores a los $2000 \mathrm{~m}$ de altitud. El riesgo es 6,4 veces mayor cuando la gestante es expuesta a la altura en el segundo trimestre ${ }^{(34)}$.

Las malformaciones más frecuentes ocurren en órganos locomotores (29\%); en la zona facial (16\%) y cardiovascular (12\%). El labio leporino y el paladar hendido son las anomalías faciales más frecuentes ${ }^{(45,46)}$. En estudios a gran escala se ha demostrado que el riesgo de labio leporino y paladar hendido está fuertemente asociado con las altura (47). En las poblaciones del sur del Perú, que parecen más adaptados a la altura (3), la tasa de malformaciones congénitas es menor ${ }^{(13)}$.

La persistencia del conducto arterioso es otra anomalía frecuente en la altura. Se considera que la hipoxemia al nacer es la causa de esta anomalía (48,49), la cual es 15-18 veces mayor en la altura ${ }^{(49)}$. La prevalencia de esta enfermedad aumenta conforme aumenta la altitud de residencia ${ }^{(50)}$.

En el ámbito fetal, la presión sanguínea de la arteria pulmonar es mayor que en la aorta, de tal manera que la sangre fluye de derecha a izquierda a través del ducto. Al nacer, la presión de la arteria pulmonar cae y se eleva la presión de la circulación sistémica y, con ello, la sangre oxigenada fluye en sentido inverso, de izquierda a derecha, generando el cierre del ducto. Este cierre es inhibido cuando la hipoxemia es exagerada como es el caso de muchos recién nacidos en la altura ${ }^{(51)}$.

El hecho que $20 \%$ de los recién nacidos presenten muy baja saturación de oxígeno al primer minuto de vida en Cerro de Pasco (4320 m de altitud) implicaría presentar mayor riesgo de padecer hipertensión arterial pulmonar; persistencia de ducto arterioso; mal de montaña crónica; entre otros ${ }^{(51)}$. Este efecto, además del Perú, se ha observado en China donde aumenta la tasa conforme aumenta la altitud ${ }^{(52)}$. También se ha observado defectos del septum auricular y ventricular ${ }^{(52,53)}$.

\section{PREECLAMPSIA}

La preeclampsia, o aumento de la presión arterial inducida por el embarazo asociado con proteinuria $(>0,3 \mathrm{~g} / 24 \mathrm{~h})$, es otro factor que puede conducir a muerte fetal tardía ${ }^{(54)}$. El riesgo aumenta con niveles de hemoglobina mayores a $14,5 \mathrm{~g} / \mathrm{dL}$ y según aumenta la altitud: a menos de 2000 m de altitud (OR: 1,73; IC95\%: 1,06-2,81); entre 2000 y $3000 \mathrm{~m}$ de altitud (OR: 1,95; IC95\%: 1,44-2,64); y más de $3000 \mathrm{~m}$ de altitud (OR: 1,42; IC95\%: 1,17-1,73) ${ }^{(55)}$. 
Es importante recalcar que estos estudios demuestran que tanto la elevación de la hemoglobina como la altura per se son factores que en forma individual pueden afectar la salud fetal ${ }^{(18,20)}$. Por cada 1000 metros de aumento de altitud, la concentración de hemoglobina aumenta en $1,52 \mathrm{~g} / \mathrm{dL}$ y el peso al nacer disminuye en $117 \mathrm{~g}^{(56)}$.

La frecuencia de muerte perinatal, bajo peso al nacer y parto pretérmino fue mayor en aquellas gestantes con valores de hemoglobina mayores a 13,2 g/dL en comparación con las que presentaron valores intermedios $(10,4-13,2 \mathrm{~g} / \mathrm{dL})$ entre las 13 y 19 semanas de gestación a nivel del mar ${ }^{(57)}$. Esto indicaría que valores altos como se encuentran en las gestantes en la altura o luego de la exposición aguda en la altura afectarían negativamente el resultado del embarazo.

De manera fisiológica la gestante, tanto a nivel del mar como en la altura, reduce sus niveles de hemoglobina en el segundo y tercer trimestre, y retorna a valores previos del embarazo al terminar este ${ }^{(20,55)}$. Esta disminución de la hemoglobina o hematocrito es debida a una expansión del volumen vascular ${ }^{(55)}$ con la finalidad de disminuir la viscosidad sanguínea y mejorar el flujo arterial úteroplacentario.

Un aumento en los niveles de hemoglobina en el embarazo puede resultar en mayor viscosidad sanguínea; disminución del flujo útero-placentario, y retardo en el crecimiento intrauterino ${ }^{(18,20,55)}$. A ello se asocia el efecto de la hipoxia propia de la altura ${ }^{(18,20)}$.

\section{SITUACIÓN DE UNA GESTANTE EXPUESTA AGUDAMENTE EN LA ALTURA}

Se describe en la literatura que las más importantes contraindicaciones absolutas y relativas para viajar a zonas de altura son entre otros, el embarazo ${ }^{(58)}$.

No existen muchos estudios referentes al efecto de la exposición aguda a la altura durante el embarazo. Un estudio en Colorado, EE.UU. (3000 m de altitud) demostró que el parto pretérmino y las hemorragias, son las complicaciones más frecuentes en las embarazadas que visitan las alturas ${ }^{(59)}$. De acuerdo con los especialistas, una exposición a alturas por encima de los $2500 \mathrm{~m}$ de altitud resulta en efectos negativos ${ }^{(60)}$. Igualmente, el ejercicio en la altura asociado al embarazo puede afectar el flujo arterial uterino comprometiendo la liberación de oxígeno al feto ${ }^{(61)}$.

Los efectos adversos de la exposición aguda a la altura es el mal de montaña agudo, el edema agudo de pulmón

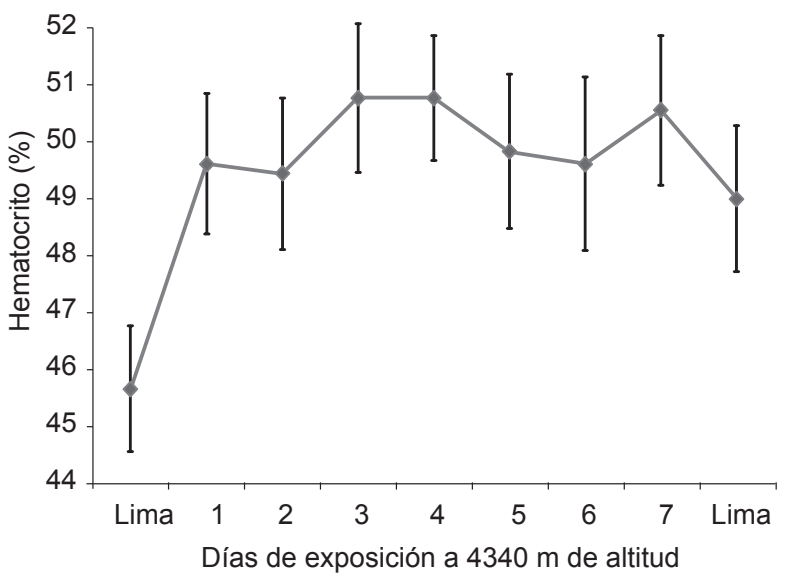

Figura 1. Cambios en el valor de hematocrito según el tiempo de exposición a la altura de Cerro de Pasco (4340 m de altitud). Fuente: Gonzales (no publicada)

y el edema agudo cerebral; estos dos últimos pueden llegar a ser mortales ${ }^{(58,62)}$.

Existen otros cambios que ocurren por efecto de la altura y que en la gestante podría tener efecto negativo. De acuerdo con nuestras investigaciones, el hematocrito se incrementa desde el primer día de exposición a la altura debido a una hemoconcentración consecuencia de la extravasación de fluidos al espacio extracelular. Este incremento en el hematocrito es exponencial a través del tiempo ${ }^{63)}$. Un típico resultado de la exposición aguda a la altura es el edema que puede ser de diversa magnitud y comportarse de manera diferente en las personas (Figura 1).

Se ha demostrado que poco después del arribo a la altura hay acumulación de fluido alveolar ${ }^{(62)}$, y esta puede ser un factor importante, además de la hipertensión pulmonar, en el edema agudo de pulmón. Ello también contribuye a la hemoconcentración y aumento de la hemoglobina/hematocrito con el consecuente daño al feto ${ }^{(18,20)}$ y la madre ${ }^{(56)}$.

Otro cambio con la exposición aguda a la altura es la disminución de la glicemia. En una exposición de 5 a 6 días a $4340 \mathrm{~m}$ de altitud, la glicemia disminuye gradualmente desde el segundo hasta el sexto día ${ }^{(64)}$. Recientemente se ha demostrado que la reducción en el crecimiento fetal en la altura estaría asociada con hipoglicemia fetal, hipoinsulinemia y tendencia a la lactacidemia ${ }^{(23)}$. Esto concuerda con la demostración que en suero, las gestantes en labor de parto en La Oroya (3800 m de altitud) muestran la mitad de los valores de insulina y seis veces menos el factor de crecimiento similar a la insulina tipo I (IGF-I). Estos autores concluyeron: Que el menor peso del recién nacido en la altura puede 
deberse a los menores niveles de IGF-I e insulina (65). Posteriores estudios muestran que a partir de la semana 25 de gestación, los niveles de la proteína ligadora de IGF-1 son mayores en la altura que a nivel del mar, lo que restringiría el crecimiento fetal ${ }^{(66)}$.

En estudios experimentales, la exposición intermitente a la hipoxia se asocia con niveles altos de hematocrito/hemoglobina y una disminución en el número de crías ${ }^{(67)}$.

\section{CONCLUSIÓN}

En conclusión, el embarazo en una mujer expuesta de forma aguda, intermitente o permanente a las grandes alturas, genera mayores riesgos para resultados adversos del embarazo que los observados si el embarazo se desarrolla a nivel del mar. Es recomendable evitar que mujeres que viven a nivel del mar desarrollen un embarazo a grandes alturas; en todo caso, deberán tener un mayor número de controles prenatales. Igualmente, se recomienda evitar el suplemento de hierro en mujeres con niveles altos de hemoglobina en la altura $(>14,5 \mathrm{~g} / \mathrm{dL})$.

\section{Fuentes de financiamiento}

Autofinanciado.

\section{Conflictos de interés}

El autor declara no tener conflictos de interés en la publicación de este artículo.

\section{REFERENCIAS BIBLIOGRÁFICAS}

1. Gonzales GF, Villena A. Aclimatación y Adaptación. En: El Futbol y la Aclimatación a la altura. Lima: Ediciones IIA; 1998. p. 23-46.

2. Hartinger S, Tapia V, Carrillo C, Bejarano L, Gonzales GF. Birth weight at high altitudes in Peru. Int J Gynaecol Obstet. 2006;93(3):275-81.

3. Gonzales GF. Peruvian contributions to the study on human reproduction at high altitude: from the chronicles of the Spanish conquest to the present. Respir Physiol Neurobiol. 2007;158(2-3):172-9.

4. McAuliffe F, Kametas N, Krampl E, Ernsting J, Nicolaides K. Blood gases in pregnancy at sea level and at high altitude. BJOG. 2001;108(9):980-5.

5. Gonzales GF, Villena A. Biomedical factors associated to high fertility at high altitude. Acta Andin. 1995;4(2):95-101.

6. Gonzales GF, Villena A, Ubilluz M. Age at menarche in Peruvian girls at sea level and at high altitude: Effect of ethnic background and socioeconomic status. Am J Hum Biol. 1996;8(4):457-63.

7. Gonzales GF, Villena A. Age at menopause in Central Andean Peruvian Women. Menopause. 1997;4:32-8.
8. Escudero F, Gonzales GF, Goñez C. Hormone profile during the menstrual cycle at high altitude. Int J Gynaecol Obstet. 1996;55(1):49-58.

9. Gonzales GF, Carrillo C. Low serum prolactin levels in women at high altitude. Int $\mathrm{J}$ Gynaecol Obstet. 1993;43(2):169-75

10. Gonzales GF, Goñez C. High serum follicle stimulating hormone (FSH) during perimenopause at high altitude. Int $\mathrm{J}$ Gynaecol Obstet. 2000;68(2):159-61.

11. De La Calancha A. Crónica Moralizadora de la Orden de San Agustín. Barcelona: Imprenta Pedro Lacaballería; 1639.

12. Martinez Vela B. Anales de la Villa Imperial de Potosí. Archivos Bolivianos - Vicente Ballivian y Rosas. Paris: Imprenta A. Franck; 1872.

13. Passano S. Características de las gestantes y de los recién nacidos en Puno a 3812 m [tesis doctoral]. Lima, Perú. Facultad de Medicina, Universidad Peruana Cayetano Heredia. 1983.

14. Cobo B. Historia del Nuevo Mundo. Madrid: Ediciones Atlas; 1964.

15. Moore LG, Niermeyer S, Zamudio S. Human adaptation to high altitude: regional and life-cycle perspectives. Am J Phys Anthropol. 1998;Suppl 27:25-64.

16. Julian CG. High altitude during pregnancy. Clin Chest Med. 2011;32(1):21-31.

17. Krampl E. Pregnancy at high altitude. Ultrasound Obstet Gynecol. 2002;19(6):535-9.

18. Gonzales GF, Tapia V, Gasco M, Carrillo CE. Maternal hemoglobin concentration and adverse pregnancy outcomes at low and moderate altitudes in Peru. J Matern Fetal Neonatal Med. 2011 Oct 17. [Epub ahead of print]

19. Moore LG, Charles SM, Julian CG. Human at high altitude: hypoxia and fetal growth. Respir Physiol Neurobiol. 2011;178(1):181-90.

20. Gonzales GF, Steenland K, Tapia V. Maternal hemoglobin level and fetal outcome at low and high altitudes. Am J Physiol Regul Integr Comp Physiol. 2009;297(5):R1477-85

21. Julian CG, Wilson MJ, Lopez M, Yamashiro H, Tellez W, Rodriguez A, et al. Augmented uterine artery blood flow and oxygen delivery protect Andeans from altitudeassociated reductions in fetal growth. Am J Physiol Regul Integr Comp Physiol. 2009;296(5):R1564-75.

22. Kametas NA, Krampl E, McAuliffe F, Rampling MW, Nicolaides KH. Pregnancy at high altitude: a hyperviscosity state. Acta Obstet Gynecol Scand. 2004;83(7):627-33.

23. Zamudio S, Torricos T, Fik E, Oyala M, Echalar L, Pullockaran $\mathbf{J}$, et al. Hypoglycemia and the origin of hypoxia-induced reduction in human fetal growth. PLoS One. 2010;5(1):e8551.

24. Gonzales GF. Metabolismo en las grandes Alturas. Acta Andina. 2001;9(1-2):31-42.

25. Soothill PW, Nicolaides $\mathrm{KH}$, Roeeck CH, Gamsu H. Blood gases and acid-base status of the human secondtrimester fetus. Obstet Gynecol 1986;68(2):173-6.

26. Postigo L, Heredia G, Illsley NP, Torricos T, Dolan C, Echalar L, et al. Where the $\mathrm{O} 2$ goes to: preservation of human fetal oxygen delivery and consumption at high altitude. J Physiol. 2009;587(Pt 3):693-708. 
27. Giussani DA, Phillips PS, Anstee S, Barker DJ. Effects of altitude versus economic status on birth weight and body shape at birth. Pediatr Res. 2001;49(4):490-4.

28. Burton GJ, Watson AL, Hempstock J, Skepper JN, Jauniaux E. Uterine glands provide histiotrophic nutrition for the human fetus during the first trimester of pregnancy. J Clin Endocrinol Metab. 2002;87(6):2954-9

29. Burton GJ, Hempstock J, Jauniaux E. Nutrition of the human fetus Turing the first trimester--- a review. Placenta. 2001;22 Suppl A:S70-7

30. Red-Horse K, Zhou Y, Genbacev O, Prakobphol A, Foulk $\mathbf{R}$, McMaster $\mathbf{M}$, et al. Trophoblast differentiation during embryo implantation and formation of the maternal-fetal interface. J Clin Invest. 2004;114(6):744-54.

31. Gonzales GF, Tapia V, Carrillo CE. Stillbirth rates in Peruvian populations at high altitude. Int J Gynaecol Obstet. 2008;100(3):221-7.

32. Jauniaux E, Watson AL, Hempstock J, Bao YP, Skepper JN, Burton GJ. Onset of maternal arterial blood flow and placental oxidative stress: a possible factor in human early pregnancy failure. Am J Pathol. 2000;157(6):2111-22.

33. Burton GJ, Jauniaux E. Placental oxidative stress: from miscarriage to preeclampsia. J Soc Gynecol Investig. 2004;11(6):342-52.

34. Alderman BW, Zamudio S, Barón AE, Joshua SC, Fernbach SK, Greene C, et al. Increased risk of craniosynostosis with higher antenatal maternal altitude. Int J Epidemiol. 1995;24(2):420-6.

35. Jensen GM, Moore LG. The effect of high altitude and other risks factors on birthweight: independent or interactive effects? Am J Pub Health. 1997;87(6):1003-7.

36. Orioli IM, Riberiro MG, Castilla EE. Clinical and epidemiological studies of amniotic deformity, adhesion, and mutilation (ADAM) sequence in a South American (ECLAM) population. Am J Med Genet A. 2003;118A(2):135-45.

37. Krampl E, Lees C, Bland JM, Espinoza Dorado J, Moscoso G, Campbell S. Fetal biometry at $4300 \mathrm{~m}$ compared to sea level in Peru. Ultrasound Obstet Gynecol. 2000;16(1):9-18.

38. Gonzales GF, Tapia V. Birth weight charts for gestational age in 63,620 healthy infants born in Peruvian public hospitals at low and at high altitude. Acta Paediatr. 2009;98(3):454-8.

39. Zacharias N. Perinatal mortality [Internet]. Walthman, MA: UpToDate; 2012 [acceso 17 de mayo de 2012]. Disponible en: http://www.uptodate.com/

40. Huiza L, Pacora P, Ayala M, Buzzio Y. La muerte fetal y la muerte neonatal tienen origen multifactorial. An Fac Med Lima. 2003;64(1):13-20.

41. Cnattingius S, Haglund B, Kramer MS. Differences in late fetal deaths rates in association with determinants of small for gestacional age fetuses: population based cohort study. BMJ. 1998;316(7143):1483-7.

42. Castilla EE, López-Camelo JJ, Campaña H. Altitude as a risk factor for congenital anomalies. Am J Med Genet. 1999;86(1):9-14.

43. López-Camelo JS, Orioli IM. Heterogeneous rates for birth defects in Latin America: hints on causality. Genet Epidemiol. 1996;13(5):469-81.

44. Webster WS, Howe AM, Abela D, Oakes DJ. The relationship between cleft lip, maxilalry hypoplasia, hypoxia and phenytoin. Curr Pharm Des. 2006;12(12):1431-48.

45. Herrera R, Li Lora JA, Ingalls TH, Marticorena E. Malformaciones congénitas del recién nacido en la altura. Arch Biol Andina 1977;7(2):94-101.

46. Silva G. Congenital malformations at high altitude: Cerro de Pasco [resumen]. Tercer Congreso Nacional de Medicina de la Altura. Cerro de Pasco, Perú. Octubre 1985, pp 97.

47. Poletta FA, Castilla EE, Orioli IM, lopez-Camelo JS. Regional analysis on the occurrence of oral clefts in South America. Am J Med Genet A. 2007;143A(24):3216-27.

48. Alzamora Castro V, Battilana G, Abugattas R, Sialer S. Patent ductus arteriosus and high altitude. Am J Cardiol. 1960;5:761-3

49. Gamboa R, Marticorena E, Peñaloza D. The ductus arteriosus in the newborn infant at high altitude. Vasa. 1972;1(3):192-5.

50. Penaloza D, Arias-Stella J, Sime F, Recavarren S, Marticorena E. The heart and pulmonary circulation in children at high altitudes: physiological, anatomical and clinical observations. Pediatrics. 1964;34:568-82.

51. Penaloza D, Arias-Stella J. The heart and pulmonary circulation at high altitudes: healthy highlanders and chronic mountain sickness. Circulation. 2007;115(9):1132-46.

52. Miao CY, Zuberbuhler JS, Zuberbuhler JR. Prevalence of congenital cardiac anomalies at high altitude. J Am Coll Cardiol. 1988;12(1):224-8.

53. Jin XH, Chen QH, Tong YF, Zhu CK, Qu Y, Zhao GQ, et al. [Study on the epidemiology of congenital heart disease in Tibetan ethnic children aged from 4 to 18 living at different altitudes in Qinghai province]. [Article in Chinese]. Zhonghua Liu Xing Bing Xue Za Zhi. 2008;29(4):317-320.

54. Facchinetti F, Alberico S, Benedetto C, Cetin I, Cozzolino S, Di Renzo GC, et al. A multicenter, case-control study on risk factors for antepartum stillbirth. J Matern Fetal Neonatal Med. 2011;24(3):407-10.

55. Gonzales GF, Tapia V, Gasco M, Carrillo CE, Fort AL. Association of hemoglobin values at booking with adverse maternal outcomes among Peruvian populations living at different altitudes. Int J Gynaecol Obstet. 2012;117(2):1349. Epub 2012 Feb 20.

56. Nahum GG, Stanislaw H. Hemoglobin, altitude and Birth weight: does maternal anemia during pregnancy influence fetal growth? J Reprod Med. 2004;49(4):297-305.

57. Murphy JF, O'Riordan J, Newcombe RG, Coles EC, Pearson JF. Relation of haemoglobin levels in first and second trimesters to outcome of pregnancy. Lancet. 1986;1(8488):992-5

58. Willems $\mathbf{J H}$. [Preconditions for a stay in high altitude areas in the case of existing health problems]. [Article in Dutch]. Ned Tijdschr Geneeskd. 2004;148(45):2216-20.

59. Niermeyer S. The pregnant altitude visitor. Adv Exp Med Biol. 1999;474:65-77.

60. Baumann H, Huch R. [Altitude exposure and staying at high altitude in pregnancy: effects on the mother and fetus]. Zentralbl Gynakol. 1986;108(15):889-99.

61. Entin PL, Coffin L. Physiological basis for recommendations regarding exercise during pregnancy at high altitude. High Alt Med Biol. 2004;5(3):321-34. 
62. Scherrer U, Rexhaj E, Jayet PY, Allemann Y, Sartori C. New insights in the pathogenesis of high-altitude pulmonary edema. Prog Cardiovasc Dis. 2010;52(6):485-92.

63. Zaobornyj T, Valdez LB, Iglesias DE, Gasco M, Gonzales GF, Boveris A. Mitochondrial nitric oxide metabolism during rat heart adaptation to high altitude: effect of sildenafil, L-NAME, and L-arginine treatments. Am J Physiol Heart Circ Physiol. 2009;296(6):H1741-7.

64. Gonzales GF, Kaneku L, Abuid J. La interrelación hormonal durante la exposición aguda a la altura de Cerro de Pasco (4340 m) en humanos. Libro de Resúmenes de las VIII Jornadas de Endocrinología. Piura, Perú Oct 28-31. 1979. p. 22 (Res 11).

65. Cipriani E, Villena AE, Martina M, Roe C, Arauco O. Niveles séricos maternos y neonatales de insulina y factores de crecimiento insulino-símiles I y II (IGF I y II) en la altura y a nivel del mar. Acta Andina 1994;3(2):145-9.
66. Krampl E, Kametas NA, McAuliffe F, Cacho-Zegarra AM, Nicolaides KH. Maternal serum insulin-like growth factor binding protein-1 in pregnancy at high altitude. Obstet Gynecol. 2002;99(4):594-8.

67. Cikutovic M, Fuentes N, Bustos-Obregón E. Effect of intermittent hypoxia on the reproduction of rats exposed to high altitude in the Chilean Altiplano. High Alt Med Biol. 2009;10(4):357-63.

Correspondencia: Gustavo F. Gonzales

Dirección: Av. Honorio Delgado 430, Lima 31, Perú.

Teléfono: (511) 3190000 anexo 2535

Correo electrónico: gustavo.gonzales@upch.pe

\section{Visite nuestra página en Facebook, www.facebook.com/rpmesp Infórmese sobre los eventos y los nuevos contenidos de la Revista Peruana de Medicina Experimental y Salud Pública}

\section{facebook \\ Buscar personas, lugares y cosas \\ Q \\ REVISTA PERUANA DE MEDICINA EXPERIMENTAL Y SALUD PÚBLICA} Difundiendo investigación en salud
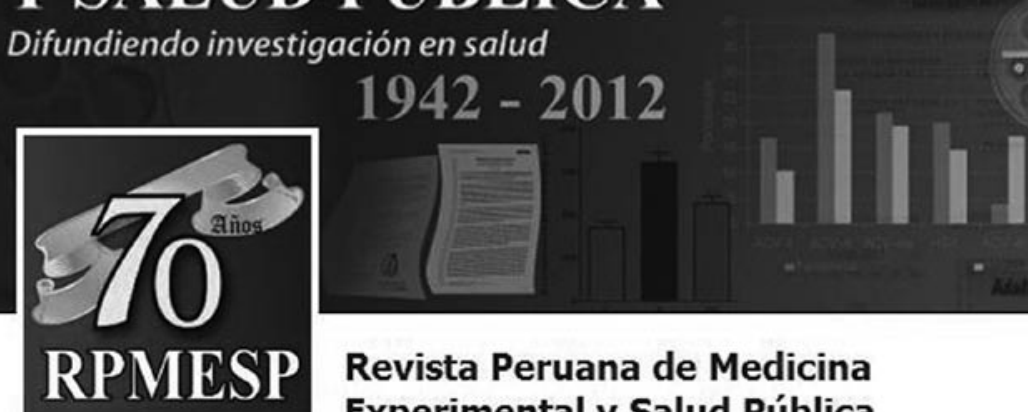

\section{Revista Peruana de Medicina Experimental y Salud Pública (OFICIAL)}

A 197 personas les gusta esta página 19 personas están hablando sobre esto

Medios de comunicaoión/Noticias/Editorial

La Revista Peruana de Medicina Experimental y Salud Publica es el órgano oficial de difusión científica del Instituto Nacional de Salud
Información

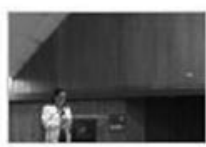

Fotos

\section{7}

Me gusta

Mensaje * -

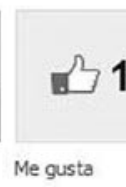

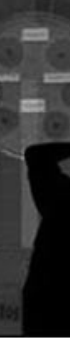

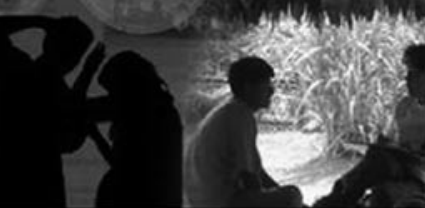

